\title{
Holographic symmetry algebras for gauge theory and gravity
}

\author{
A. Guevara, ${ }^{a, b}$ E. Himwich, ${ }^{a}$ M. Pate ${ }^{a, b}$ and A. Strominger ${ }^{a}$ \\ ${ }^{a}$ Center for the Fundamental Laws of Nature, Harvard University, \\ 17 Oxford Street, Cambridge, MA 02138, U.S.A. \\ ${ }^{b}$ Society of Fellows, Harvard University, \\ 78 Mount Auburn Street, Cambridge, MA 02138, U.S.A. \\ E-mail: aguevaragonzalez@fas.harvard.edu, himwich@g.harvard.edu, \\ mpate@g.harvard.edu, strominger@physics.harvard.edu
}

ABSTRACT: All 4D gauge and gravitational theories in asymptotically flat spacetimes contain an infinite number of non-trivial symmetries. They can be succinctly characterized by generalized 2D currents acting on the celestial sphere. A complete classification of these symmetries and their algebras is an open problem. Here we construct two towers of such 2D currents from positive-helicity photons, gluons, or gravitons with integer conformal weights. These generate the symmetries associated to an infinite tower of conformally soft theorems. The current algebra commutators are explicitly derived from the poles in the OPE coefficients, and found to comprise a rich closed subalgebra of the complete symmetry algebra.

Keywords: Global Symmetries, Scattering Amplitudes, Space-Time Symmetries, Higher Spin Symmetry

ARXiv EPRINT: 2103.03961 


\section{Contents}

1 Introduction 1

2 Gluons 3

3 Gravitons $\quad 6$

4 Gluons and gravitons $\quad 7$

$\begin{array}{lll}5 & \text { OPE from scattering amplitudes } & 7\end{array}$

$\begin{array}{llr}6 & \text { Summary } & 10\end{array}$

A Gluon OPE calculation $\quad 11$

B The OPE conformal block 12

\section{Introduction}

A central question in physics is: "What are the fundamental symmetries of nature?" One aspect of this question motivates the search for Beyond-the-Standard-Model physics and Unification. A second aspect, and the focus of the present work, is fully characterizing the non-trivial symmetries inherent in the laws of physics that have already been verified experimentally: namely, General Relativity (GR) and the Standard Model.

It is surprising that we still don't have a complete answer to this question, or even a precise formulation of the question itself. GR has a diffeomorphism symmetry, but this is really a redundancy of description, often referred to as a "trivial" symmetry. Of greater interest are "non-trivial" symmetries which have, by Noether's theorem, associated conservation laws with measurable consequences, such as conservation of linear/angular momentum or boost charge. One might have suspected that the Poincaré symmetries of Special Relativity - which imply these conservation laws - are the only non-trivial symmetries of GR (in the asymptotically flat context considered here). Famously, BMS [1, 2] showed in 1962 that this could not be the case, and consequently that there is no limit in which General reduces to Special Relativity. Only very recently [3, 4], using soft theorems from quantum field theory, was it shown that there are an infinite number of non-trivial symmetries of GR with associated conserved charges. These comprise a subgroup of the symmetries considered by BMS and can be measured using the gravitational memory effect [5]. However, various developments [6-14] have made it clear that these are not all of the non-trivial symmetries of GR. Currently, there is not even a proposal for a complete classification of 
the non-trivial symmetries of nature! For QED the situation is similarly unresolved [15-23]. There is active research on this topic from a variety of viewpoints [24-61].

There are many ways to characterize symmetries, which should ultimately all be equivalent. A familiar and traditional method is the canonical construction of symmetry generators as conserved charges that commute with the Hamiltonian or the $\mathcal{S}$-matrix. ${ }^{1}$ Other methods directly derive relations among $\mathcal{S}$-matrix elements, such as those given by soft theorems, or assume falloffs and perform an asymptotic symmetry analysis. An especially powerful, recently-developed "celestial" approach employs the holographic reformulation of the $4 \mathrm{D} \mathcal{S}$-matrix as a $2 \mathrm{D}$ conformal correlator on the celestial sphere at null infinity. In this approach, non-trivial symmetries correspond to generalized conformal currents on the celestial sphere. Their properties and algebra can be efficiently computed using the constraints of 2D conformal invariance. A further advantage is that one largely avoids ambiguities associated to gauge choices, boundary terms, and falloff conditions. This paper takes a step towards classifying the non-trivial symmetries of nature within this approach.

The symmetry-generating currents are of two types, arising from positive or negative helicity conformally soft massless particles. Whenever two opposite-helicity soft particles are scattered, the result can depend on the order of soft limits and a prescription of some kind is required to define the $\mathcal{S}$-matrix. In this paper we sidestep this important issue by considering only positive helicity currents ${ }^{2}$ and working in a $\operatorname{Vir}_{L} \otimes \operatorname{SL}(2, \mathbb{R})_{R}$-invariant formalism. ${ }^{3}$ Consistent with this restriction, this paper reports on a tower of higher-spin symmetry generators forming a closed generalized current-algebra sector of the celestial $\mathrm{CFT}_{2}$. The symmetries we find are only a subgroup of all of the symmetries in gravity and gauge theory, but a large and interesting one.

This infinite tower of symmetries is likely related to the infinite tower of soft theorems that have been discussed in the literature $[23,63,64]$. We will show, however, that commutators of the leading, subleading, and (in gravity) subsubleading symmetries generate the whole tower, so the new symmetries here give no new constraints on the $\mathcal{S}$-matrix.

In practice our discussion is largely for tree-level Einstein-Yang-Mills theory, but the methods are generally applicable and we anticipate that the algebra persists in some form in the presence of quantum corrections and arbitrary higher-dimension operators coming from UV physics. The results for gluons are affected if IR confinement occurs. The algebra is also affected by a short list of higher-dimension operators $[65,66]$ which deform the subleading soft theorems.

The pure gluon algebra is derived in section 2. In $4 \mathrm{D}$ nonabelian gauge theory with group $G$, the leading soft theorem implies a standard closed 2D celestial $G$-current algebra $[24,38,43,46,67]$. The subleading soft theorem implies two further $G$-valued

\footnotetext{
${ }^{1}$ We consider here the asymptotically flat approximation in which the cosmological constant vanishes and there is an $\mathcal{S}$-matrix.

${ }^{2}$ As noted in [56], this restriction arises automatically when considering the MHV sector, in which one helicity decouples.

${ }^{3}$ Throughout this paper we treat left and right movers as independent on the celestial sphere, which means we effectively work in $(2,2)$ signature, i.e. Klein space [62]. The $\operatorname{SL}(2, \mathbb{R})_{R}$ here is the global subgroup of the $\operatorname{Vir}_{R}$ superrotations.
} 
holomorphic currents $[18,44,56]$. We show that these form an $\operatorname{SL}(2, \mathbb{R})_{R}$ doublet. Commutators of two of these currents give yet further symmetry generators, which form an $\operatorname{SL}(2, \mathbb{R})_{R}$ triplet. Continuing in this manner, we construct an infinite tower of $G$-currents in finite-dimensional $\mathrm{SL}(2, \mathbb{R})_{R}$ representations and present their algebra. These currents have integral left+right conformal dimensions $\Delta=1,0,-1, \ldots$ where the operator product expansion is known to contain poles [44, 48]. Spacetime translation invariance, which is not manifest in this presentation, combines these currents into a representation of the Poincaré group.

In section 3, we consider the algebra of conformally soft positive helicity gravitons. Here

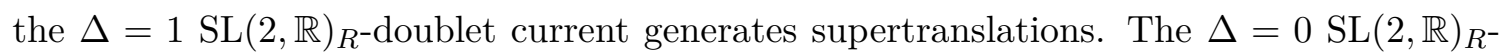
triplet current closes with itself. ${ }^{4}$ At $\Delta=-1$ one encounters the $\operatorname{SL}(2, \mathbb{R})_{R}$-quadruplet current associated to the subsubleading soft graviton theorem. These three currents then generate an infinite tower of currents, whose algebra we present. In section 4 we couple gluons and gravitons and determine the resulting algebra. A directly analogous result applies to the coupling between photons and gravitons, although we do not include explicit formulae for that case.

In section 5, we show explicitly that the OPEs we derive are encoded in four-gluon MHV scattering amplitudes, a result that can be extended to general multiplicity using the BCFW construction as described in [48]. Section 6 collects our results for the full $\mathrm{SL}(2, \mathbb{R})_{R}$ gluon and graviton algebra and we describe the straightforward application of these results to photons. Appendix A contains details of the gluon-gluon OPE calculation, which the graviton-graviton and gluon-graviton OPE calculations closely mimic. Appendix B presents a compact, manifestly conformally covariant formula for the contribution to an OPE from a primary and all its $\mathrm{SL}(2, \mathbb{R})_{L} \otimes \mathrm{SL}(2, \mathbb{R})_{R}$ descendants. While this work was in progress $[55,56]$ appeared with overlapping results.

\section{Gluons}

In this section, we find a class of positive helicity gluon operators $O_{\Delta}^{a,+}(z, \bar{z})$ at special conformal weights that generate symmetries of the $\mathcal{S}$-matrix. ${ }^{5}$ Our starting point is the leading OPE for two conformal primary, positive helicity gluons:

$$
O_{\Delta_{1}}^{a,+}\left(z_{1}, \bar{z}_{1}\right) O_{\Delta_{2}}^{b,+}\left(z_{2}, \bar{z}_{2}\right) \sim \frac{-i f^{a b}{ }^{c}}{z_{12}} B\left(\Delta_{1}-1, \Delta_{2}-1\right) O_{\Delta_{1}+\Delta_{2}-1}^{c,+}\left(z_{2}, \bar{z}_{2}\right),
$$

where $z_{12}=z_{1}-z_{2}$ and $B(x, y)=\frac{\Gamma(x) \Gamma(y)}{\Gamma(x+y)}$ is the Euler beta function. This expression was derived from a Mellin transform of the known soft collinear gluon singularities

\footnotetext{
${ }^{4}$ The shadow of this current is the $\operatorname{Vir}_{R}$ stress tensor in [27].

${ }^{5}$ Following the conventions of [44], $O_{\Delta}^{a, \pm}$ denotes an outgoing conformal primary gluon of conformal (or boost) weight $\Delta=h+\bar{h}$, adjoint group index $a$, and helicity $s= \pm 1$. The group structure constants obey the Jacobi identity

$$
f^{a b}{ }_{d} f^{d c e}+f^{b c}{ }_{d} f^{d a e}+f^{c a}{ }_{d} f^{d b e}=0 .
$$

Details of the map from the momentum-space $\mathcal{S}$-matrix to celestial correlation functions are included in section 5 .
} 
in [38] and from symmetries in [44]. It is considerably less complicated than the general OPE [44] that involves both helicities of gluons as well as gravitons. ${ }^{6}$ A salient feature of (2.1) is the infinite sequence of poles in the OPE coefficient at integral conformal weights $\Delta_{1}=k=1,0,-1, \ldots$. As we shall see, the fact that the weights are typically negative leads to some interesting and perhaps unfamiliar structures. The highest weight case with $\Delta=1$ corresponds to the conformally soft gluon current and turns out to be holomorphic. Contour integrals of this current on the celestial sphere generate the large gauge symmetries of the $\mathcal{S}$-matrix [67]. The case $\Delta=0$ is the subleading soft current, which lies in an $\operatorname{SL}(2, \mathbb{R})_{R}$ doublet with negative $\bar{h}=-\frac{1}{2} \cdot{ }^{7}$ Contour integrals of these currents generate subleading soft symmetries $[18,21]$. The properties of the rest of the operators with integral $\Delta<0$, which generate an infinite tower of soft theorems, have not been well understood. Here we will construct the $2 \mathrm{D}$ algebra of these currents and find that it is closed. This tower is likely not yet the complete celestial symmetry algebra, which may also include Goldstone currents $[14,22,32,60]$ and negative helicity gluon and graviton generators or their shadows. However, it is an interesting and non-trivial subalgebra thereof.

A description of the algebra that is manifestly covariant under the full $\operatorname{Vir}_{L} \otimes \operatorname{Vir}_{R}$ $2 \mathrm{D}$ conformal group is challenging. Here we cut the problem down to manageable size by presenting the algebra in a manifestly $\operatorname{Vir}_{L} \otimes \operatorname{SL}(2, \mathbb{R})_{R^{-c o v a r i a n t}}$ form. This is natural because the positive helicity symmetry currents fall into finite $(2-k)$-dimensional $\mathrm{SL}(2, \mathbb{R})_{R}$ representations with $\bar{h}=\frac{k-1}{2}$ and highest (lowest) weights $\frac{1-k}{2}\left(\frac{k-1}{2}\right) .{ }^{8}$ The tower starts with (and we will see is generated by) the leading $k=1$ and subleading $k=0$ soft currents, which are a singlet and doublet, respectively. Mode-expanding on the right

$$
O_{k}^{a,+}(z, \bar{z})=\sum_{n} \frac{O_{k, n}^{a,+}(z)}{\bar{z}^{n+\frac{k-1}{2}}},
$$

conformally soft currents are defined by ${ }^{9}$

$$
R_{n}^{k, a}(z):=\lim _{\varepsilon \rightarrow 0} \varepsilon O_{k+\varepsilon, n}^{a,+}(z), \quad k=1,0,-1,-2, \ldots, \quad \frac{k-1}{2} \leq n \leq \frac{1-k}{2} .
$$

For future notational convenience we also define

$$
R^{k, a}(z, \bar{z})=\sum_{n=\frac{k-1}{2}}^{\frac{1-k}{2}} \frac{R_{n}^{k, a}(z)}{\bar{z}^{n+\frac{k-1}{2}}},
$$

which has weights

$$
(h, \bar{h})=\left(\frac{k+1}{2}, \frac{k-1}{2}\right) .
$$

\footnotetext{
${ }^{6}$ Corrections to this OPE from higher-dimension operators are suppressed by positive powers of $z_{12}$ or $\bar{z}_{12}$ and would largely not affect the following analysis [44]. The one interesting exception is $F^{3}$, which produces a potentially relevant term proportional $\frac{\bar{z}_{12}^{2}}{z_{12}} O_{\Delta_{1}+\Delta_{2}+1}^{a,-}$.

${ }^{7}$ The zero mode of the leading $\Delta=1$ current generates global color rotations, which are a global symmetry of the vacuum. This is the only unbroken global symmetry: all higher modes of the leading current are spontaneously broken, as well as the all modes of the further subleading currents.

${ }^{8}$ This is equivalent to the null states found in [56].

${ }^{9}$ Outside the specified range of $n$, the $\mathrm{SL}(2, \mathbb{R})_{R}$-invariant norm vanishes. Such operators may still have contact interactions but in this paper operators are always at distinct points.
} 
These values of conformal weights $\Delta=k$ include all the conformally soft poles encountered in the OPE (2.1). The factor of $\varepsilon$ incorporated in (2.3) is needed to cancel these poles, leading to finite OPEs for the rescaled $R^{k, a}$.

Using e.g. conformal blocks (see OPE blocks from [68], reviewed in appendix B) to derive the contribution from $\operatorname{SL}(2, \mathbb{R})_{R}$ descendants, the OPE (2.1) has the further expansion in $\bar{z}_{12}$

$$
O_{\Delta_{1}}^{a,+}\left(z_{1}, \bar{z}_{1}\right) O_{\Delta_{2}}^{b,+}\left(z_{2}, \bar{z}_{2}\right) \sim \frac{-i f^{a b} c}{z_{12}} \sum_{n=0}^{\infty} B\left(\Delta_{1}-1+n, \Delta_{2}-1\right) \frac{\left(\bar{z}_{12}\right)^{n}}{n !} \bar{\partial}^{n} O_{\Delta_{1}+\Delta_{2}-1}^{c,+}\left(z_{2}, \bar{z}_{2}\right) .
$$

The OPE of the conformally soft gluon operators (2.4) then becomes

$$
R^{k, a}\left(z_{1}, \bar{z}_{1}\right) R^{l, b}\left(z_{2}, \bar{z}_{2}\right) \sim \frac{-i f_{c}^{a b}{ }_{c}^{1-k}}{z_{12}} \sum_{n=0}\left(\begin{array}{c}
2-k-l-n \\
1-l
\end{array}\right) \frac{\left(\bar{z}_{12}\right)^{n}}{n !} \bar{\partial}^{n} R^{k+l-1, c}\left(z_{2}, \bar{z}_{2}\right),
$$

where in truncating the sum we make use of $\bar{\partial}^{2-k} R^{k, a}=0$. We can consider derivatives

$$
\bar{\partial}^{p} R^{k, a}\left(z_{1}, \bar{z}_{1}\right) \bar{\partial}^{q} R^{l, b}\left(z_{2}, \bar{z}_{2}\right) \sim \frac{-i f_{c}^{a b}}{z_{12}}\left(\begin{array}{c}
2-k-l-p-q \\
1-l-q
\end{array}\right) \bar{\partial}^{q+p} R^{k+l-1, c}\left(z_{2}, \bar{z}_{2}\right),
$$

where $0 \leq p<2-k$ and $0 \leq q<2-l$ and we now include only leading order terms in both $z_{12}$ and $\bar{z}_{12}$. Defining the commutator for holomorphic objects ${ }^{10}$

$$
[A, B](z)=\oint_{z} \frac{d w}{2 \pi i} A(w) B(z)
$$

and mode-expanding on the right, (2.8) can be reexpressed

$$
\left[R_{n}^{k, a}, R_{n^{\prime}}^{l, b}\right]=-i f^{a b}{ }_{c}\left(\begin{array}{c}
\frac{1-k}{2}-n+\frac{1-l}{2}-n^{\prime} \\
\frac{1-k}{2}-n
\end{array}\right)\left(\begin{array}{c}
\frac{1-k}{2}+n+\frac{1-l}{2}+n^{\prime} \\
\frac{1-k}{2}+n
\end{array}\right) R_{n+n^{\prime}}^{k+l-1, c} .
$$

This is the general conformally soft gluon algebra. For details of the derivation, see appendix A. One may verify directly that this expression satisfies the Jacobi identity and is translation covariant. ${ }^{11}$

The lowest-weight $\mathrm{SL}(2, \mathbb{R})_{R}$ element $R_{\frac{k-1}{2}}^{k, a}(z)$ for each $k$ is annihilated by $\bar{L}_{-1}=i \bar{\partial}$, and hence is holomorphic. These operators have an especially simple chiral algebra. Define

$$
\widehat{R}^{k, a}(z):=\bar{\partial}^{1-k} R^{k, a}(z, \bar{z})=(1-k) ! R_{\frac{k-1}{2}}^{k, a}(z) .
$$

Setting $p=1-k$ and $q=1-l$ in (2.8), we obtain the relation

$$
\widehat{R}^{k, a}\left(z_{1}\right) \widehat{R}^{l, b}\left(z_{2}\right) \sim \frac{-i f^{a b}{ }_{c}}{z_{12}} \widehat{R}^{k+l-1, c}\left(z_{2}\right) .
$$

\footnotetext{
${ }^{10}$ Note that this is a $2 \mathrm{D}$ celestial commutator on a $1 \mathrm{D}$ circle, not to be mistaken for a $4 \mathrm{D}$ commutator on a $3 \mathrm{D}$ slice.

${ }^{11}$ Translation covariance follows from the Jacobi identity with conformally soft gravitons which are studied in subsequent sections. See for example (4.3).
} 
Equivalently,

$$
\left[\widehat{R}^{k, a}, \widehat{R}^{l, b}\right]=-i f^{a b}{ }_{c} \widehat{R}^{k+l-1, c} .
$$

Hence the algebra of the $\operatorname{SL}(2, \mathbb{R})_{R}$ lowest-weight states considerably simplifies, as does that of the $\operatorname{SL}(2, \mathbb{R})_{R}$ highest-weight states. Most of the complexity in (2.10) arises from the $\operatorname{SL}(2, \mathbb{R})_{R}$ mode level structure.

\section{Gravitons}

The analysis for positive-helicity gravitons $G_{\Delta}^{+}(z, \bar{z})$ is analogous to that for gluons. ${ }^{12}$ Define a family of conformally soft positive-helicity gravitons

$$
H^{k}=\lim _{\varepsilon \rightarrow 0} \varepsilon G_{k+\varepsilon}^{+}, \quad k=2,1,0,-1, \ldots,
$$

with weights

$$
(h, \bar{h})=\left(\frac{k+2}{2}, \frac{k-2}{2}\right),
$$

and a consistently truncated antiholomorphic mode expansion,

$$
H^{k}(z, \bar{z})=\sum_{n=\frac{k-2}{2}}^{\frac{2-k}{2}} \frac{H_{n}^{k}(z)}{\bar{z}^{n+\frac{k-2}{2}}} .
$$

The $k=1$ term generates supertranslations. Expanding $H_{n}^{1}(z)=\sum_{m} H_{m, n}^{1} z^{-m-3 / 2}$, the four modes $H_{ \pm \frac{1}{2}, \pm \frac{1}{2}}^{1}$ generate the four global translations.

The OPE of two conformal primary gravitons of arbitrary weight [44], including antiholomorphic descendants, is

$$
G_{\Delta_{1}}^{+}\left(z_{1}, \bar{z}_{1}\right) G_{\Delta_{2}}^{+}\left(z_{2}, \bar{z}_{2}\right) \sim-\frac{\kappa}{2} \frac{1}{z_{12}} \sum_{n=0}^{\infty} B\left(\Delta_{1}-1+n, \Delta_{2}-1\right) \frac{\left(\bar{z}_{12}\right)^{n+1}}{n !} \bar{\partial}^{n} G_{\Delta_{1}+\Delta_{2}}^{+}\left(z_{2}, \bar{z}_{2}\right),
$$

with $\kappa=\sqrt{32 \pi G}$. The OPE of conformally soft gravitons (3.1) becomes

$$
H^{k}\left(z_{1}, \bar{z}_{1}\right) H^{l}\left(z_{2}, \bar{z}_{2}\right) \sim-\frac{\kappa}{2} \frac{1}{z_{12}} \sum_{n=0}^{1-k}\left(\begin{array}{c}
2-k-l-n \\
1-l
\end{array}\right) \frac{\left(\bar{z}_{12}\right)^{n+1}}{n !} \bar{\partial}^{n} H^{k+l}\left(z_{2}, \bar{z}_{2}\right) .
$$

After some algebra (analogous to the gluon case in appendix A) one finds the current commutators

$$
\left[H_{m}^{k}, H_{n}^{l}\right]=-\frac{\kappa}{2}[n(2-k)-m(2-l)] \frac{\left(\frac{2-k}{2}-m+\frac{2-l}{2}-n-1\right) !}{\left(\frac{2-k}{2}-m\right) !\left(\frac{2-l}{2}-n\right) !} \frac{\left(\frac{2-k}{2}+m+\frac{2-l}{2}+n-1\right) !}{\left(\frac{2-k}{2}+m\right) !\left(\frac{2-l}{2}+n\right) !} H_{m+n}^{k+l} .
$$

As in the gauge theory case, we check that this commutator obeys the Jacobi identity with three $H$ operators. These imply the $H$ operators obey the Jacobi identity with $\bar{L}_{0}, \bar{L}_{ \pm 1}$, and that it is translation covariant.

\footnotetext{
${ }^{12}$ Again following [44], $G_{\Delta}^{ \pm}(z, \bar{z})$ denotes an outgoing conformal primary graviton of weight $\Delta$ and helicity $s= \pm 2$.
} 
For the case of gluons, there is a closed subalgebra with $k=1$. For gravitons, the closed subalgebra has $k=0$. Defining

$$
J_{1}=-\frac{2}{\kappa} H_{1}^{0}, \quad J_{0}=\frac{1}{\kappa} H_{0}^{0}, \quad J_{-1}=-\frac{2}{\kappa} H_{-1}^{0},
$$

one finds from (3.6) the current algebra

$$
\left[J_{m}, J_{n}\right]=(m-n) J_{m+n}
$$

The zero modes of $J_{m}$ generate self-dual Lorentz transformations in Klein space.

\section{Gluons and gravitons}

As in the previous sections, we begin with the OPE of conformally soft gluons and gravitons of arbitrary weight derived in [44], and include antiholomorphic descendants:

$$
G_{\Delta_{1}}^{+}\left(z_{1}, \bar{z}_{1}\right) O_{\Delta_{2}}^{a,+}\left(z_{2}, \bar{z}_{2}\right) \sim-\frac{\kappa}{2} \frac{1}{z_{12}} \sum_{n=0}^{\infty} B\left(\Delta_{1}+n-1, \Delta_{2}\right) \frac{\bar{z}_{12}^{n+1}}{n !} \bar{\partial}^{n} O_{\Delta_{1}+\Delta_{2}}^{a,+}\left(z_{2}, \bar{z}_{2}\right) .
$$

In terms of the conformally soft gluons and gravitons defined above, the OPE becomes

$$
H^{k}\left(z_{1}, \bar{z}_{1}\right) R^{l, a}\left(z_{2}, \bar{z}_{2}\right) \sim-\frac{\kappa}{2} \frac{1}{z_{12}} \sum_{n=0}^{1-k} \frac{(1-k-l-n) !}{(1-k-n) !(-l) !} \frac{\bar{z}_{12}^{n+1}}{n !} \bar{\partial}^{n} R^{k+l, a}\left(z_{2}, \bar{z}_{2}\right),
$$

where we again make use of the finite $\mathrm{SL}(2, \mathbb{R})_{R}$ representation to truncate the sum. After a calculation analogous to that in previous sections, we derive the commutator

$$
\left[H_{m}^{k}, R_{n}^{l, a}\right]=-\frac{\kappa}{2}[n(2-k)-m(1-l)] \frac{\left(\frac{2-k}{2}-m+\frac{1-l}{2}-n-1\right) !}{\left(\frac{2-k}{2}-m\right) !\left(\frac{1-l}{2}-n\right) !} \frac{\left(\frac{2-k}{2}+m+\frac{1-l}{2}+n-1\right) !}{\left(\frac{2-k}{2}+m\right) !\left(\frac{1-l}{2}+n\right) !} R_{m+n}^{k+l, a} .
$$

\section{OPE from scattering amplitudes}

In order to make contact with other work on scattering amplitudes, we will show how the previous OPEs including all antiholomorphic descendants emerge when the celestial primaries are identified with scattering states in MHV tree amplitudes (see also [54, 69]). In momentum space, massless $n$-particle amplitudes $\mathbf{A}\left(\epsilon_{i} \omega_{i}, z_{i}\right)$ are labeled by energies $\omega_{i}$ and points $z_{i}$ on the celestial sphere. This follows from a parametrization of massless momenta

$$
p_{k}^{\mu}=\frac{\epsilon_{k} \omega_{k}}{\sqrt{2}}\left(1+z_{k} \bar{z}_{k}, z_{k}+\bar{z}_{k},-i\left(z_{k}-\bar{z}_{k}\right), 1-z_{k} \bar{z}_{k}\right),
$$

with $\epsilon_{k}= \pm 1$ for outgoing and incoming momenta, respectively. Celestial amplitudes $\mathcal{A}\left(\Delta_{i}, z_{i}\right)$ are defined by transforming to Mellin space (see e.g. [70, 71])

$$
\mathcal{A}_{s_{j}}\left(\Delta_{j}, z_{j}\right)=\left(\prod_{i=1}^{n} \int_{0}^{\infty} \frac{d \omega_{i}}{\omega_{i}} \omega_{i}^{\Delta_{i}}\right) \mathbf{A}_{s_{i}}\left(\epsilon_{i} \omega_{i}, z_{i}\right)
$$


where $s_{i}$ are helicity labels. ${ }^{13}$ Celestial amplitudes are naturally interpreted as correlation functions of $n$ weight $\left(h_{i}, \bar{h}_{i}\right)=\left(\frac{\Delta_{i}+s_{i}}{2}, \frac{\Delta_{i}-s_{i}}{2}\right)$ conformal primary operators on the celestial sphere:

$$
\mathcal{A}_{s_{i}}\left(\Delta_{i}, z_{i}\right) \rightarrow\left\langle\mathcal{O}_{\Delta_{1}}^{s_{1}}\left(z_{1}, \bar{z}_{1}\right) \cdots \mathcal{O}_{\Delta_{n}}^{s_{n}}\left(z_{n}, \bar{z}_{n}\right)\right\rangle
$$

Let us consider first the case of gluons with $s_{i}= \pm 1$. Nicely, the full OPE (2.6) is encoded even in the simplest non-trivial scattering process, namely $n=4$ gluons. We start from

$$
\begin{aligned}
\mathbf{A}_{--++}^{a b c d}\left(\left\{\epsilon_{1} \omega_{1}, z_{1}, \bar{z}_{1}\right\}, \ldots,\left\{\epsilon_{4} \omega_{4}, z_{4}, \bar{z}_{4}\right\}\right)= & \delta^{4}\left(\sum_{i=1}^{4} \epsilon_{i} \lambda_{i}^{\alpha} \tilde{\lambda}_{i}^{\dot{\alpha}}\right) \times \\
& {\left[f^{a d}{ }_{e} f^{c b e} \frac{\langle 12\rangle^{3}}{\langle 23\rangle\langle 34\rangle\langle 41\rangle}+f^{a c}{ }_{e} f^{d b e} \frac{\langle 12\rangle^{3}}{\langle 24\rangle\langle 43\rangle\langle 31\rangle}\right], }
\end{aligned}
$$

with null momenta written as $p_{\alpha \dot{\alpha}}=\epsilon \lambda_{\alpha} \tilde{\lambda}_{\dot{\alpha}}$ using

$$
\lambda_{i}=\sqrt{\omega_{i}}\left(\begin{array}{ll}
1 & z_{i}
\end{array}\right), \tilde{\lambda}_{i}=\sqrt{\omega_{i}}\left(\begin{array}{c}
1 \\
\bar{z}_{i}
\end{array}\right)
$$

where $\langle i j\rangle=\epsilon_{i} \epsilon_{j} \lambda_{i}^{\alpha} \lambda_{j}^{\beta} \epsilon_{\alpha \beta}$. We will consider the holomorphic collinear limit for positivehelicity gluons 3,4 , which we take as outgoing $\epsilon_{3}=\epsilon_{4}=+1$. To study only the antiholomorphic descendants, using the parametrization (5.5), we can extract the leading order behavior as $z_{3} \rightarrow z_{4}$ while keeping the exact dependence on $\bar{z}_{34}$ :

$$
\begin{aligned}
\delta^{4}\left(\sum_{i=1}^{4} \epsilon_{i} \lambda_{i}^{\alpha} \tilde{\lambda}_{i}^{\dot{\alpha}}\right)= & \frac{1}{\omega_{1} \omega_{2} z_{12}^{2}} \delta\left(\epsilon_{1} \omega_{1}-\frac{z_{24}}{z_{12}}\left(\omega_{3}+\omega_{4}\right)\right) \delta\left(\epsilon_{2} \omega_{2}-\frac{z_{41}}{z_{12}}\left(\omega_{3}+\omega_{4}\right)\right) \delta\left(\bar{z}_{14}-\epsilon_{1} \frac{\omega_{3}}{\omega_{1}} \frac{z_{24}}{z_{12}} \bar{z}_{34}\right) \\
& \times \delta\left(\bar{z}_{24}-\epsilon_{2} \frac{\omega_{3}}{\omega_{2}} \frac{z_{41}}{z_{12}} \bar{z}_{34}\right)+\mathcal{O}\left(z_{34}\right) .
\end{aligned}
$$

Introducing $\omega_{3}=t \omega, \omega_{4}=(1-t) \omega$ with $0 \leq t \leq 1$, this becomes

$$
\begin{array}{r}
\frac{1}{\omega_{1} \omega_{2} z_{12}^{2}} \delta\left(\epsilon_{1} \omega_{1}-\frac{z_{24}}{z_{12}} \omega\right) \delta\left(\epsilon_{2} \omega_{2}-\frac{z_{41}}{z_{12}} \omega\right) \delta\left(\bar{z}_{14}-t \bar{z}_{34}\right) \delta\left(\bar{z}_{24}-t \bar{z}_{34}\right) \\
=\left.\delta^{4}\left(\sum_{i=1,2,4} \epsilon_{i} \lambda_{i}^{\alpha} \tilde{\lambda}_{i}^{\dot{\alpha}}\right)\right|_{\bar{z}_{4} \rightarrow \bar{z}_{4}+t \bar{z}_{34}, \omega_{4} \rightarrow \omega},
\end{array}
$$

i.e. at leading order in $z_{34}$, the $n=4$ momentum conservation condition can be written as the $n=3$ condition together with the deformation

$$
\bar{z}_{4} \rightarrow \bar{z}_{4}+t \bar{z}_{34}
$$

This deformation extends trivially to the full amplitude (5.4) since the remaining factor does not depend on the antiholomorphic coordinates. Indeed, at leading order in $z_{34} \sim\langle 34\rangle$ the stripped part of (5.4) becomes

$$
f^{a d} f_{e}^{c b e} \frac{\langle 12\rangle^{3}}{\langle 23\rangle\langle 34\rangle\langle 41\rangle}+f^{a c} f_{e}^{d b e} \frac{\langle 12\rangle^{3}}{\langle 24\rangle\langle 43\rangle\langle 31\rangle} \rightarrow \frac{f^{c d} e_{e}}{z_{34} \omega t(1-t)} \times\left(f^{a b e} \frac{\langle 12\rangle^{3}}{\langle 24\rangle\langle 41\rangle}\right)_{\omega_{4} \rightarrow \omega},
$$

\footnotetext{
${ }^{13}$ Mellin amplitudes converge only for certain $\Delta_{i}$ but can be defined generally by analytic continuation $[60,72]$.
} 
and we can relate the full $n=4$ amplitude to an $n=3$ amplitude:

$$
\begin{aligned}
\mathbf{A}_{--++}^{a b c d} & \left(\left\{\epsilon_{1} \omega_{1}, z_{1}, \bar{z}_{1}\right\},\left\{\epsilon_{2} \omega_{2}, z_{2}, \bar{z}_{2}\right\},\left\{\omega_{3}, z_{3}, \bar{z}_{3}\right\},\left\{\omega_{4}, z_{4}, \bar{z}_{4}\right\}\right) \\
& =\frac{-i f^{c d}}{z_{34} \omega t(1-t)} \mathbf{A}_{--+}^{a b e}\left(\left\{\epsilon_{1} \omega_{1}, z_{1}, \bar{z}_{1}\right\},\left\{\epsilon_{2} \omega_{2}, z_{2}, \bar{z}_{2}\right\},\left\{\omega, z_{4}, \bar{z}_{4}+t \bar{z}_{34}\right\}\right)+\mathcal{O}\left(z_{34}^{0}\right) .
\end{aligned}
$$

To translate the above statement into a celestial correlation function we employ (5.2)-(5.3) and find

$$
\begin{aligned}
& \left\langle O_{\Delta_{1}}^{a,-} \cdots O_{\Delta_{4}}^{d,+}\right\rangle \\
& =\int d \omega_{1} \omega_{1}^{\Delta_{1}-1} d \omega_{2} \omega_{2}^{\Delta_{2}-1} d \omega \omega^{\Delta_{3}+\Delta_{4}-1} d t t^{\Delta_{3}-1}(1-t)^{\Delta_{4}-1} \mathbf{A}_{--++}^{a b c d} \\
& \rightarrow \frac{-i f{ }^{c d} e}{z_{34}} \int d t t^{\Delta_{3}-2}(1-t)^{\Delta_{4}-2} \\
& \quad \times \int d \omega_{1} \omega_{1}^{\Delta_{1}-1} d \omega_{2} \omega_{2}^{\Delta_{2}-1} d \omega \omega^{\Delta_{3}+\Delta_{4}-2} \mathbf{A}_{--+}^{a b e}\left(\left\{-\omega_{1}, z_{1}, \bar{z}_{1}\right\},\left\{-\omega_{2}, z_{2}, \bar{z}_{2}\right\},\left\{\omega, z_{4}, \bar{z}_{4}+t \bar{z}_{34}\right\}\right) \\
& =\frac{-i f f_{e}}{z_{34}} \int d t t^{\Delta_{3}-2}(1-t)^{\Delta_{4}-2}\left\langle O_{\Delta_{1}}^{a,-}\left(z_{1}, \bar{z}_{1}\right) O_{\Delta_{2}}^{b,-}\left(z_{2}, \bar{z}_{2}\right) O_{\Delta_{3}+\Delta_{4}-1}^{e,+}\left(z_{4}, \bar{z}_{4}+t \bar{z}_{34}\right)\right\rangle,
\end{aligned}
$$

where gluons 1,2 are incoming and 3,4 are outgoing. The OPE of two positive helicity gluons follows:

$$
O_{\Delta_{3}}^{c,+}\left(z_{3}, \bar{z}_{3}\right) O_{\Delta_{4}}^{d,+}\left(z_{4}, \bar{z}_{4}\right) \sim \frac{-i f_{e}^{c d}}{z_{34}} \int d t t^{\Delta_{3}-2}(1-t)^{\Delta_{4}-2} O_{\Delta_{3}+\Delta_{4}-1}^{e,+}\left(z_{4}, \bar{z}_{4}+t \bar{z}_{34}\right) .
$$

This expression corresponds to a conformal block including all antiholomorphic descendants. This connection is detailed in appendix B. To see this explicitly we perform a Taylor expansion in $\bar{z}_{34}$ :

$$
\begin{aligned}
O_{\Delta_{3}}^{c,+}\left(z_{3}, \bar{z}_{3}\right) O_{\Delta_{4}}^{d,+}\left(z_{4}, \bar{z}_{4}\right) & \sim \frac{-i f^{c d} e}{z_{34}} \sum_{n=0}^{\infty} \int d t t^{\Delta_{3}-2+n}(1-t)^{\Delta_{4}-2} \frac{\bar{z}_{34}^{n}}{n !} \bar{\partial}^{n} O_{\Delta_{3}+\Delta_{4}-1}^{e,+}\left(z_{4}, \bar{z}_{4}\right) \\
& \sim \frac{-i f^{c d} e}{z_{34}} \sum_{n=0}^{\infty} B\left(\Delta_{3}-1+n, \Delta_{4}-1\right) \frac{\bar{z}_{34}^{n}}{n !} \bar{\partial}^{n} O_{\Delta_{3}+\Delta_{4}-1}^{e,+}\left(z_{4}, \bar{z}_{4}\right),
\end{aligned}
$$

in agreement with (2.6). For gravitons, analogous computations hold for the $n=4 \mathrm{MHV}$ celestial correlators. For instance, the four-graviton amplitude in [45] leads to the conformal block

$$
G_{\Delta_{3}}^{+}\left(z_{3}, \bar{z}_{3}\right) G_{\Delta_{4}}^{+}\left(z_{4}, \bar{z}_{4}\right) \sim-\frac{\kappa}{2} \frac{\bar{z}_{34}}{z_{34}} \int d t t^{\Delta_{3}-2}(1-t)^{\Delta_{4}-2} G_{\Delta_{3}+\Delta_{4}}^{+}\left(z_{4}, \bar{z}_{4}+t \bar{z}_{34}\right),
$$

which can be Taylor expanded to obtain (3.4).

To see that the above gluon-gluon OPE is consistent with scattering amplitudes at any multiplicity and general helicity configuration, we generalize the expression (5.10) as follows:

$$
\begin{aligned}
\mathbf{A}_{\left\{s_{j}\right\}++}^{\left\{a_{j}\right\} c d}\left(\left\{\omega_{1}, z_{1}, \bar{z}_{1}\right\}\right. & \left.\ldots,\left\{\omega_{n+1}, z_{n+1}, \bar{z}_{n+1}\right\}\right) \\
& \sim \frac{-i f^{c d}}{z_{n, n+1} \omega t(1-t)} \mathbf{A}_{\left\{s_{j}\right\}+}^{\left\{a_{j}\right\} e}\left(\left\{\omega_{1}, z_{1}, \bar{z}_{1}\right\}, \ldots,\left\{\omega, z_{n}, \bar{z}_{n}+t \bar{z}_{n+1, n}\right\}\right)
\end{aligned}
$$


where $s_{j}= \pm 1, j=1, \ldots, n-1$ and we have set again $\omega_{n+1}=t \omega, \omega_{n}=(1-t) \omega$. It is then straightforward, following the steps of eq. (5.11), to show that the OPE (5.12) follows.

The formula (5.15) can be proven from the BCFW recurrence relations of gluon scattering amplitudes, following the construction of [48] (see also references therein). The $n+1$-point scattering amplitude obtained by attaching a positive helicity gluon of coordinates $\left\{\omega_{s}, z_{s}, \bar{z}_{s}\right\}$ is written as

$$
\begin{aligned}
& \mathbf{A}_{\left\{s_{j}\right\}+}^{\left\{a_{j}\right\} a_{s}}\left(\left\{\omega_{1}, z_{1}, \bar{z}_{1}\right\}, \ldots,\left\{\omega_{s}, z_{s}, \bar{z}_{s}\right\}\right) \\
& \sim \sum_{i=2}^{n}-i f^{a_{s} a_{i}} b \frac{z_{1 i}}{z_{1 s} z_{s i} \omega_{s}}\left(1+\alpha_{i}\right)^{s_{i}}\left(1+\beta_{i}\right)^{s_{1}} \times \\
& \quad \mathbf{A}_{\left\{s_{j}\right\}}^{\left\{a_{j}, a_{i} \rightarrow b\right\}}\left(\left\{\left(1+\beta_{i}\right) \omega_{1}, z_{1}, \frac{\bar{z}_{1}+\beta_{i} \bar{z}_{s}}{1+\beta_{i}}\right\}, \ldots,\left\{\left(1+\alpha_{i}\right) \omega_{i}, z_{i}, \frac{\bar{z}_{i}+\alpha_{i} \bar{z}_{s}}{1+\alpha_{i}}\right\}, \ldots\right)
\end{aligned}
$$

where $\alpha_{i}=\frac{\omega_{s} z_{1 s}}{\omega_{i} z_{1 i}}, \beta_{i}=\frac{\omega_{s} z_{i s}}{\omega_{1} z_{i 1}}$ and we have neglected, following [48], multiparticle factorizations which are regular in the OPE limit. Let us again consider the leading behaviour as $z_{n} \rightarrow z_{s}$, keeping $\bar{z}_{n s}$ finite. For this we need only keep the term $i=n$ in the above sum and set $s_{n}=+1$ for the positive helicity gluon. Introducing $\omega_{s}=t \omega, \omega_{n}=(1-t) \omega$ as anticipated we find $\alpha_{n} \rightarrow \frac{t}{1-t}, \beta_{n} \rightarrow 0$ and the leading behaviour of (5.16) becomes precisely (5.15).

\section{Summary}

We collect the results for the conformally soft algebra derived in the previous sections:

$$
\begin{aligned}
{\left[R_{n}^{k, a}, R_{n^{\prime}}^{l, b}\right] } & =-i f_{c}^{a b} \frac{\left(\frac{1-k}{2}-n+\frac{1-l}{2}-n^{\prime}\right) !}{\left(\frac{1-k}{2}-n\right) !\left(\frac{1-l}{2}-n^{\prime}\right) !} \frac{\left(\frac{1-k}{2}+n+\frac{1-l}{2}+n^{\prime}\right) !}{\left(\frac{1-k}{2}+n\right) !\left(\frac{1-l}{2}+n^{\prime}\right) !} R_{n+n^{\prime}}^{k+l-1, c}, \\
{\left[H_{n}^{k}, H_{n^{\prime}}^{l}\right] } & =-\frac{\kappa}{2}\left[n^{\prime}(2-k)-n(2-l)\right] \frac{\left(\frac{2-k}{2}-n+\frac{2-l}{2}-n^{\prime}-1\right) !}{\left(\frac{2-k}{2}-n\right) !\left(\frac{2-l}{2}-n^{\prime}\right) !} \frac{\left(\frac{2-k}{2}+n+\frac{2-l}{2}+n^{\prime}-1\right) !}{\left(\frac{2-k}{2}+n\right) !\left(\frac{2-l}{2}+n^{\prime}\right) !} H_{n+n^{\prime}}^{k+l}, \\
{\left[H_{n}^{k}, R_{n^{\prime}}^{l, a}\right] } & =-\frac{\kappa}{2}\left[n^{\prime}(2-k)-n(1-l)\right] \frac{\left(\frac{2-k}{2}-n+\frac{1-l}{2}-n^{\prime}-1\right) !}{\left(\frac{2-k}{2}-n\right) !\left(\frac{1-l}{2}-n^{\prime}\right) !} \frac{\left(\frac{2-k}{2}+n+\frac{1-l}{2}+n^{\prime}-1\right) !}{\left(\frac{2-k}{2}+n\right) !\left(\frac{1-l}{2}+n^{\prime}\right) !} R_{n+n^{\prime}}^{k+l, a} .
\end{aligned}
$$

These results readily extend to photons. In particular, one can construct generalized conformally soft photon currents that are directly analogous to the generalized conformally soft gluon currents. Unlike gluons, which carry color, photons do not carry electric charge and as a result their generalized currents commute with one another. However, since photons couple to gravitons, the photon currents obey commutation relations with the graviton currents that are of the same form as the gluon-graviton commutation relations.

\section{Acknowledgments}

We are grateful to Alex Atanasov, Adam Ball, Dan Kapec, Walker Melton, and Ana Raclariu for useful conversations. This work was supported by DOE grant de-sc/0007870. AG and MP are supported by Junior Fellowships at the Harvard Society of Fellows. 


\section{A Gluon OPE calculation}

The OPE of generalized soft gluon operators $R^{k, a}$ is derived from the OPE of conformal primary gluons

$$
O_{\Delta_{1}}^{a,+}\left(z_{1}, \bar{z}_{1}\right) O_{\Delta_{2}}^{b,+}\left(z_{2}, \bar{z}_{2}\right) \sim \frac{-i f^{a b} c}{z_{12}} \sum_{n=0}^{\infty} B\left(\Delta_{1}-1+n, \Delta_{2}-1\right) \frac{\bar{z}_{12}^{n}}{n !} \partial_{\bar{z}_{2}}^{n} O_{\Delta_{1}+\Delta_{2}-1}^{c,+}\left(z_{2}, \bar{z}_{2}\right),
$$

using the definition

$$
R^{k, a}(z, \bar{z})=\lim _{\varepsilon \rightarrow 0} \varepsilon O_{k+\varepsilon}^{a,+}(z, \bar{z}), \quad k=1,0,-1,-2, \cdots
$$

with the mode expansion

$$
R^{k, a}(z, \bar{z})=\sum_{n=\frac{k-1}{2}}^{\frac{1-k}{2}} \frac{R_{n}^{k, a}(z)}{\bar{z}^{n+\frac{k-1}{2}}}
$$

Taking the simultaneous limit of (A.1), we find

$$
\begin{aligned}
R^{k, a}\left(z_{1}, \bar{z}_{1}\right) R^{\ell, b}\left(z_{2}, \bar{z}_{2}\right) & =\lim _{\varepsilon \rightarrow 0} \varepsilon O_{k+\varepsilon}^{a,+}\left(z_{1}, \bar{z}_{1}\right) \varepsilon O_{\ell+\varepsilon}^{b,+}\left(z_{2}, \bar{z}_{2}\right) \\
& \sim \frac{-i f_{c}^{a b}}{z_{12}} \sum_{m=0}^{1-k} \frac{1}{m !} \frac{(2-k-\ell-m) !}{(1-k-m) !(1-\ell) !} \bar{z}_{12}^{m} \partial_{\bar{z}_{2}}^{m} R^{k+\ell-1, c}\left(z_{2}, \bar{z}_{2}\right),
\end{aligned}
$$

where the truncation in the sum over $m$ follows from the mode expansion (A.3). (Specifically, note that the powers of $\bar{z}_{1}$ match on either side of the equation.)

To determine the algebra of modes $R_{n}^{k, a}$ from the OPE (A.4), we first recall that modes are extracted from

$$
R_{n}^{k, a}(z)=\oint \frac{d \bar{z}}{2 \pi i} \bar{z}^{n+\frac{k-3}{2}} R^{k, a}(z, \bar{z}) .
$$

Here $z$ and $\bar{z}$ are treated independently, as, for example, is done in section 6.1 of [73]. Then, the algebra of modes $R_{n}^{k, a}$ is obtained by taking the following contour integrals:

$$
\left[R_{n}^{k, a}, R_{n^{\prime}}^{\ell, b}\right]\left(z_{2}\right)=\oint_{\left|\bar{z}_{1}\right|<\epsilon} \frac{d \bar{z}_{1}}{2 \pi i} \bar{z}_{1}^{n+\frac{k-3}{2}} \oint_{\left|\bar{z}_{2}\right|<\epsilon} \frac{d \bar{z}_{2}}{2 \pi i} \bar{z}_{2}^{n^{\prime}+\frac{\ell-3}{2}} \oint_{\left|z_{12}\right|<\epsilon} \frac{d z_{1}}{2 \pi i} R^{k, a}\left(z_{1}, \bar{z}_{1}\right) R^{\ell, b}\left(z_{2}, \bar{z}_{2}\right) .
$$

Note that since the OPE (A.4) is not singular in the antiholomorphic variables, the order in which contour integrals in antiholomorphic variables is taken does not matter. Let's take the $\bar{z}_{1}$ contour first. Substituting the OPE (A.4) into the right-hand side of (A.6), performing the $z_{1}$ integral and using

$$
\oint_{\left|\bar{z}_{1}\right|<\epsilon} \frac{d \bar{z}_{1}}{2 \pi i} \bar{z}_{1}^{n+\frac{k-3}{2}} \bar{z}_{12}^{m}=\left\{\begin{array}{cc}
0, & 0 \leq m<\frac{1-k}{2}-n \\
\frac{m !}{\left(\frac{1-k}{2}-n\right) !\left(m+n+\frac{k-1}{2}\right) !}\left(-\bar{z}_{2}\right)^{m+n+\frac{k-1}{2}}, & \frac{1-k}{2}-n \leq m \leq 1-k
\end{array},\right.
$$


to perform the $\bar{z}_{1}$ integral, (A.6) becomes

$$
\begin{aligned}
{\left[R_{n}^{k, a}, R_{n^{\prime}}^{\ell, b}\right]\left(z_{2}\right)=-i f_{c}^{a b} \sum_{m=\frac{1-k}{2}-n}^{1-k} } & \frac{(2-k-\ell-m) !}{(1-k-m) !(1-\ell) !} \frac{(-1)^{m+n+\frac{k-1}{2}}}{\left(\frac{1-k}{2}-n\right) !\left(m+n+\frac{k-1}{2}\right) !} \\
& \times \oint_{\left|\bar{z}_{2}\right|<\epsilon} \frac{d \bar{z}_{2}}{2 \pi i} \bar{z}_{2}^{m+n+\frac{k-1}{2}+n^{\prime}+\frac{\ell-3}{2}} \partial_{\bar{z}_{2}}^{m} R^{k+\ell-1, c}\left(z_{2}, \bar{z}_{2}\right) .
\end{aligned}
$$

To perform the remaining contour integral, we substitute $R^{k+\ell-1, c}\left(z_{2}, \bar{z}_{2}\right)$ for its mode expansion

$$
\begin{aligned}
\oint_{\left|\bar{z}_{2}\right|<\epsilon} \frac{d \bar{z}_{2}}{2 \pi i} \bar{z}_{2}^{m+n+\frac{k-1}{2}+n^{\prime}+\frac{\ell-3}{2}} \partial_{\bar{z}_{2}}^{m} R^{k+\ell-1, c}\left(z_{2}, \bar{z}_{2}\right) \\
=\oint_{\left|\bar{z}_{2}\right|<\epsilon} \frac{d \bar{z}_{2}}{2 \pi i} \bar{z}_{2}^{m+\frac{k-1}{2}+n+n^{\prime}+\frac{\ell-3}{2}} \partial_{\bar{z}_{2}}^{m} \sum_{m^{\prime}=\frac{k+\ell-2}{2}}^{\frac{2-k-\ell}{2}} \frac{R_{m^{\prime}}^{k+\ell-1, c}\left(z_{2}\right)}{\bar{z}_{2}^{m^{\prime}+\frac{k+\ell-2}{2}}} \\
=\frac{\left(\frac{1-k}{2}-n+\frac{1-\ell}{2}-n^{\prime}\right) !}{\left(\frac{1-k}{2}-n+\frac{1-\ell}{2}-n^{\prime}-m\right) !} R_{n+n^{\prime}}^{k+\ell-c}\left(z_{2}\right) .
\end{aligned}
$$

Finally, substituting this result back in (A.8) and performing the sum in $m$, we find

$$
\left[R_{n}^{k, a}, R_{n^{\prime}}^{\ell, b}\right]\left(z_{2}\right)=-i f^{a b}{ }_{c} \frac{\left(\frac{1-k}{2}-n+\frac{1-\ell}{2}-n^{\prime}\right) !}{\left(\frac{1-k}{2}-n\right) !\left(\frac{1-\ell}{2}-n^{\prime}\right) !} \frac{\left(\frac{1-k}{2}+n+\frac{1-\ell}{2}+n^{\prime}\right) !}{\left(\frac{1-k}{2}+n\right) !\left(\frac{1-\ell}{2}+n^{\prime}\right) !} R_{n+n^{\prime}}^{k+\ell-1, c}\left(z_{2}\right) .
$$

\section{B The OPE conformal block}

In this appendix we will derive the integral expression (5.12) encoding the contribution from all $\operatorname{SL}(2, \mathbb{R})_{R}$ descendants. To do so we first start with the complete expression for $\operatorname{SL}(2, \mathbb{R})_{L} \otimes \mathrm{SL}(2, \mathbb{R})_{R}$ descendants as given in e.g. appendix $\mathrm{B}$ of $[68]{ }^{14}$ Consider two primaries of weights $\left(h_{1}, \bar{h}_{1}\right),\left(h_{2}, \bar{h}_{2}\right)$. In the shadow representation, singling out the contribution from a single primary $P$ and all its descendants leads to the expression

$$
\begin{aligned}
& O_{\left(h_{1}, \bar{h}_{1}\right)}\left(z_{1}, \bar{z}_{1}\right) O_{\left(h_{2}, \bar{h}_{2}\right)}\left(z_{2}, \bar{z}_{2}\right)
\end{aligned}
$$

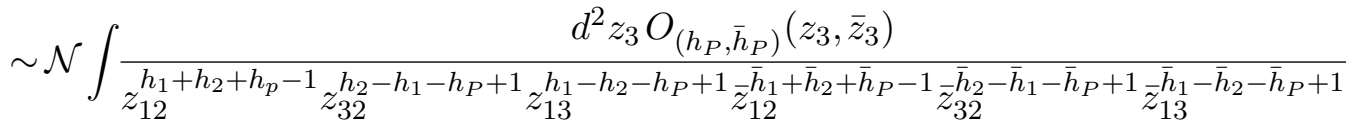

$$
\begin{aligned}
& =\mathcal{N} I_{O_{1} O_{2}}^{O_{3}} \text {, }
\end{aligned}
$$

where $\mathcal{N}$ is a normalization constant that can be fixed by comparing leading order terms in the limit $z_{12}, \bar{z}_{12} \rightarrow 0$. As coordinates $z, \bar{z}$ are independent we will assume they both lie on the real projective line. If $z_{1}>z_{2}, \bar{z}_{1}>\bar{z}_{2}$, the KLT formula ${ }^{15}$ provides a factorization

\footnotetext{
${ }^{14}$ Recall we are working with independent left and right coordinates, i.e. $(2,2)$ signature [62].

${ }^{15}$ To make connection with the standard four-point KLT formula as described in [74, 75], it is convenient to set $z_{1}=1, z_{2}=0$ using $\mathrm{SL}(2, \mathbb{R})$ covariance and expand $O_{\left(h_{P}, \bar{h}_{P}\right)}\left(z_{3}, \bar{z}_{3}\right)$ in powers of $z_{3}, \bar{z}_{3}$.
} 
of the above integral into two disk integrals:

$$
\begin{aligned}
& I_{O_{1} O_{2}}^{O_{3}}=2 \sin \left(\pi\left(h_{2}-h_{1}+h_{P}\right)\right) \int_{z_{1}}^{\infty} \frac{d z_{3}}{z_{12}^{h_{1}+h_{2}+h_{p}-1} z_{32}^{h_{2}-h_{1}-h_{P}+1} z_{31}^{h_{1}-h_{2}-h_{P}+1}}
\end{aligned}
$$

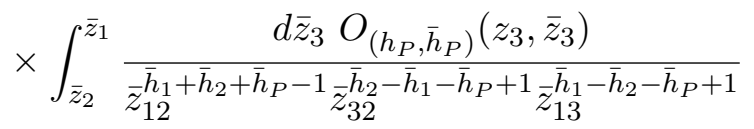

which has explicit $\mathrm{SL}(2, \mathbb{R})_{L} \otimes \mathrm{SL}(2, \mathbb{R})_{R}$ covariance. To recover the set of right descendants we perform an expansion of the left factor to leading order in $z_{12}$ by setting $z_{3}=z_{2}+t z_{12}$. We obtain

$$
\begin{aligned}
I_{O_{1} O_{2}}^{O_{3}}= & \frac{2 \sin \left(\pi\left(h_{2}-h_{1}+h_{P}\right)\right)}{z_{12}^{h_{1}+h_{2}-h_{P}}} \int_{1}^{\infty} d t t^{h_{1}+h_{P}-h_{2}-1}(t-1)^{h_{2}+h_{P}-h_{1}-1} \\
& \times \int_{\bar{z}_{2}}^{\bar{z}_{1}} \frac{d \bar{z}_{3} O_{\left(h_{P}, \bar{h}_{P}\right)}\left(z_{2}+t z_{12}, \bar{z}_{3}\right)}{\bar{z}_{12}^{\bar{h}_{1}+\bar{h}_{2}+\bar{h}_{P}-1} \bar{z}_{32}-\bar{h}_{1}-\bar{h}_{P}+1} \bar{z}_{13}^{\bar{h}_{1}-\bar{h}_{2}-\bar{h}_{P}+1} \\
& \quad \times \frac{2 \pi \Gamma\left(1-2 h_{P}\right)}{\Gamma\left(1+h_{2}-h_{1}-h_{P}\right) \Gamma\left(1+h_{1}-h_{2}-h_{P}\right) z_{12}^{h_{1}+h_{2}-h_{P}}} \\
& +\mathcal{O}\left(z_{12}^{-h_{1}-h_{2}+h_{P}+1}\right) .
\end{aligned}
$$

We thus identify the remaining real integral as a conformal block for $\operatorname{SL}(2, \mathbb{R})_{R}$ descendants. It can be written in a more compact way by introducing $\bar{z}_{3}=\bar{z}_{2}+t \bar{z}_{12}$ :

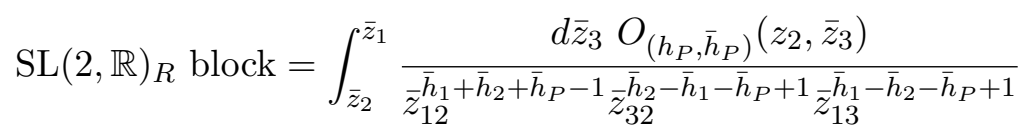

$$
\begin{aligned}
& =\frac{1}{\bar{z}_{12} \bar{h}_{2}+\bar{h}_{1}-\bar{h}_{P}} \int_{0}^{1} \frac{d t O_{\left(h_{P}, \bar{h}_{P}\right)}\left(z_{2}, \bar{z}_{2}+t \bar{z}_{12}\right)}{t^{\bar{h}_{2}-\bar{h}_{1}-\bar{h}_{P}+1}(1-t)^{\bar{h}_{1}-\bar{h}_{2}-\bar{h}_{P}+1}} .
\end{aligned}
$$

Let us apply this formula to the positive-helicity gluon case, for which $(h, \bar{h})=\left(\frac{\Delta+1}{2}, \frac{\Delta-1}{2}\right)$ and $\Delta_{P}=\Delta_{1}+\Delta_{2}-1$. We thus find

$$
I_{O_{1} O_{2}}^{O_{3}, c}=\frac{2 \pi \Gamma\left(1-\Delta_{1}-\Delta_{2}\right)}{\Gamma\left(1-\Delta_{1}\right) \Gamma\left(1-\Delta_{2}\right)} \times \frac{1}{z_{12}} \int_{0}^{1} \frac{d t O_{\Delta_{P}}^{c,+}\left(z_{2}, \bar{z}_{2}+t \bar{z}_{12}\right)}{t^{2-\Delta_{1}}(1-t)^{2-\Delta_{2}}}+\mathcal{O}\left(z_{12}^{0}\right) .
$$

The normalization factor $\mathcal{N}$ can be fixed in this case as

$$
\begin{aligned}
O_{\Delta_{1}}^{a,+} O_{\Delta_{2}}^{b,+} & \sim-i f^{a b}{ }_{c} \frac{\Gamma\left(1-\Delta_{1}\right) \Gamma\left(1-\Delta_{2}\right)}{2 \pi \Gamma\left(1-\Delta_{1}-\Delta_{2}\right)} I_{O_{1} O_{2}}^{O_{3}, c} \\
& =\frac{-i f^{a b}{ }_{c}}{z_{12}} \int_{0}^{1} \frac{d t O_{\Delta_{P}}^{c,+}\left(z_{2}, \bar{z}_{2}+t \bar{z}_{12}\right)}{t^{2-\Delta_{1}}(1-t)^{2-\Delta_{2}}}
\end{aligned}
$$

in agreement with (5.12). 
Open Access. This article is distributed under the terms of the Creative Commons Attribution License (CC-BY 4.0), which permits any use, distribution and reproduction in any medium, provided the original author(s) and source are credited.

\section{References}

[1] H. Bondi, M. van der Burg and A. Metzner, Gravitational Waves in General Relativity. 7. Waves from Axisymmetric Isolated Systems, Proc. Roy. Soc. Lond. A 269 (1962) 21.

[2] R. Sachs, Gravitational Waves in General Relativity. 8. Waves in Asymptotically Flat Space-times, Proc. Roy. Soc. Lond. A 270 (1962) 103.

[3] A. Strominger, On BMS Invariance of Gravitational Scattering, JHEP 07 (2014) 152 [arXiv:1312.2229] [INSPIRE].

[4] T. He, V. Lysov, P. Mitra and A. Strominger, BMS supertranslations and Weinberg's soft graviton theorem, JHEP 05 (2015) 151 [arXiv: 1401.7026] [INSPIRE].

[5] A. Strominger and A. Zhiboedov, Gravitational Memory, BMS Supertranslations and Soft Theorems, JHEP 01 (2016) 086 [arXiv:1411.5745] [INSPIRE].

[6] G. Barnich and C. Troessaert, BMS charge algebra, JHEP 12 (2011) 105 [arXiv:1106.0213] [INSPIRE].

[7] F. Cachazo and A. Strominger, Evidence for a New Soft Graviton Theorem, arXiv: 1404.4091 [INSPIRE].

[8] D. Kapec, V. Lysov, S. Pasterski and A. Strominger, Semiclassical Virasoro symmetry of the quantum gravity $\mathcal{S}$-matrix, JHEP 08 (2014) 058 [arXiv:1406.3312] [INSPIRE]

[9] M. Campiglia and A. Laddha, Asymptotic symmetries and subleading soft graviton theorem, Phys. Rev. D 90 (2014) 124028 [arXiv:1408.2228] [INSPIRE].

[10] M. Campiglia and A. Laddha, New symmetries for the Gravitational S-matrix, JHEP 04 (2015) 076 [arXiv: 1502.02318] [inSPIRE].

[11] S. Choi and R. Akhoury, Magnetic soft charges, dual supertranslations, and 't Hooft line dressings, Phys. Rev. D 102 (2020) 025001 [arXiv:1912.02224] [InSPIRE].

[12] H. Godazgar, M. Godazgar and C.N. Pope, New dual gravitational charges, Phys. Rev. D 99 (2019) 024013 [arXiv:1812.01641] [INSPIRE].

[13] U. Kol and M. Porrati, Properties of Dual Supertranslation Charges in Asymptotically Flat Spacetimes, Phys. Rev. D 100 (2019) 046019 [arXiv: 1907.00990] [inSPIRE].

[14] E. Himwich, S.A. Narayanan, M. Pate, N. Paul and A. Strominger, The Soft $\mathcal{S}$-Matrix in Gravity, JHEP 09 (2020) 129 [arXiv:2005.13433] [INSPIRE].

[15] T. He, P. Mitra, A.P. Porfyriadis and A. Strominger, New Symmetries of Massless QED, JHEP 10 (2014) 112 [arXiv:1407.3789] [InSPIRE].

[16] D. Kapec, M. Pate and A. Strominger, New Symmetries of QED, Adv. Theor. Math. Phys. 21 (2017) 1769 [arXiv:1506.02906] [INSPIRE].

[17] A. Strominger, Magnetic Corrections to the Soft Photon Theorem, Phys. Rev. Lett. 116 (2016) 031602 [arXiv: 1509.00543] [INSPIRE].

[18] V. Lysov, S. Pasterski and A. Strominger, Low's Subleading Soft Theorem as a Symmetry of QED, Phys. Rev. Lett. 113 (2014) 111601 [arXiv:1407.3814] [INSPIRE]. 
[19] M. Campiglia and A. Laddha, Asymptotic symmetries of QED and Weinberg's soft photon theorem, JHEP 07 (2015) 115 [arXiv: 1505.05346] [INSPIRE].

[20] M. Campiglia and A. Laddha, Subleading soft photons and large gauge transformations, JHEP 11 (2016) 012 [arXiv: 1605.09677] [INSPIRE].

[21] E. Himwich and A. Strominger, Celestial current algebra from Low's subleading soft theorem, Phys. Rev. D 100 (2019) 065001 [arXiv:1901.01622] [inSPIRE].

[22] A. Nande, M. Pate and A. Strominger, Soft Factorization in QED from 2D Kac-Moody Symmetry, JHEP 02 (2018) 079 [arXiv: 1705.00608] [INSPIRE].

[23] M. Campiglia and A. Laddha, Asymptotic charges in massless QED revisited: A view from Spatial Infinity, JHEP 05 (2019) 207 [arXiv:1810.04619] [INSPIRE].

[24] A. Strominger, Asymptotic Symmetries of Yang-Mills Theory, JHEP 07 (2014) 151 [arXiv: 1308.0589] [INSPIRE].

[25] T.T. Dumitrescu, T. He, P. Mitra and A. Strominger, Infinite-dimensional fermionic symmetry in supersymmetric gauge theories, JHEP 08 (2021) 051 [arXiv:1511.07429] [INSPIRE].

[26] S. Pasterski, Asymptotic Symmetries and Electromagnetic Memory, JHEP 09 (2017) 154 [arXiv: 1505.00716] [INSPIRE].

[27] D. Kapec, P. Mitra, A.-M. Raclariu and A. Strominger, 2D Stress Tensor for $4 D$ Gravity, Phys. Rev. Lett. 119 (2017) 121601 [arXiv:1609.00282] [INSPIRE].

[28] M. Campiglia and A. Laddha, Sub-subleading soft gravitons and large diffeomorphisms, JHEP 01 (2017) 036 [arXiv:1608.00685] [INSPIRE].

[29] S. Pasterski, S.-H. Shao and A. Strominger, Gluon Amplitudes as 2d Conformal Correlators, Phys. Rev. D 96 (2017) 085006 [arXiv:1706.03917] [InSPIRE].

[30] D. Kapec, M. Perry, A.-M. Raclariu and A. Strominger, Infrared Divergences in QED, Revisited, Phys. Rev. D 96 (2017) 085002 [arXiv:1705.04311] [INSPIRE].

[31] S. Choi and R. Akhoury, BMS Supertranslation Symmetry Implies Faddeev-Kulish Amplitudes, JHEP 02 (2018) 171 [arXiv:1712.04551] [INSPIRE].

[32] L. Donnay, A. Puhm and A. Strominger, Conformally Soft Photons and Gravitons, JHEP 01 (2019) 184 [arXiv: 1810.05219] [INSPIRE].

[33] A. Ashtekar, M. Campiglia and A. Laddha, Null infinity, the BMS group and infrared issues, Gen. Rel. Grav. 50 (2018) 140 [arXiv:1808. 07093] [INSPIRE].

[34] M. Campiglia and R. Eyheralde, Asymptotic U(1) charges at spatial infinity, JHEP 11 (2017) 168 [arXiv:1703.07884] [INSPIRE].

[35] A. Schreiber, A. Volovich and M. Zlotnikov, Tree-level gluon amplitudes on the celestial sphere, Phys. Lett. B 781 (2018) 349 [arXiv:1711.08435] [InSPIRE].

[36] S. Stieberger and T.R. Taylor, Strings on Celestial Sphere, Nucl. Phys. B 935 (2018) 388 [arXiv: 1806. 05688] [INSPIRE].

[37] S. Stieberger and T.R. Taylor, Symmetries of Celestial Amplitudes, Phys. Lett. B 793 (2019) 141 [arXiv: 1812.01080] [INSPIRE].

[38] W. Fan, A. Fotopoulos and T.R. Taylor, Soft Limits of Yang-Mills Amplitudes and Conformal Correlators, JHEP 05 (2019) 121 [arXiv: 1903.01676] [INSPIRE]. 
[39] A. Fotopoulos, S. Stieberger, T.R. Taylor and B. Zhu, Extended BMS Algebra of Celestial CFT, JHEP 03 (2020) 130 [arXiv: 1912.10973] [INSPIRE].

[40] A. Fotopoulos and T.R. Taylor, Primary Fields in Celestial CFT, JHEP 10 (2019) 167 [arXiv: 1906.10149] [INSPIRE].

[41] W. Fan, A. Fotopoulos, S. Stieberger and T.R. Taylor, On Sugawara construction on Celestial Sphere, JHEP 09 (2020) 139 [arXiv: 2005.10666] [INSPIRE].

[42] A. Fotopoulos, S. Stieberger, T.R. Taylor and B. Zhu, Extended Super BMS Algebra of Celestial CFT, JHEP 09 (2020) 198 [arXiv: 2007.03785] [INSPIRE].

[43] M. Pate, A.-M. Raclariu and A. Strominger, Conformally Soft Theorem in Gauge Theory, Phys. Rev. D 100 (2019) 085017 [arXiv: 1904.10831] [INSPIRE].

[44] M. Pate, A.-M. Raclariu, A. Strominger and E.Y. Yuan, Celestial operator products of gluons and gravitons, Rev. Math. Phys. 33 (2021) 2140003 [arXiv: 1910.07424] [InSPIRE].

[45] A. Puhm, Conformally Soft Theorem in Gravity, JHEP 09 (2020) 130 [arXiv:1905. 09799] [INSPIRE].

[46] T. Adamo, L. Mason and A. Sharma, Celestial amplitudes and conformal soft theorems, Class. Quant. Grav. 36 (2019) 205018 [arXiv: 1905.09224] [INSPIRE].

[47] D. Nandan, A. Schreiber, A. Volovich and M. Zlotnikov, Celestial Amplitudes: Conformal Partial Waves and Soft Limits, JHEP 10 (2019) 018 [arXiv:1904.10940] [INSPIRE].

[48] A. Guevara, Notes on Conformal Soft Theorems and Recursion Relations in Gravity, arXiv: 1906.07810 [INSPIRE].

[49] Y.T.A. Law and M. Zlotnikov, Poincaré constraints on celestial amplitudes, JHEP 03 (2020) 085 [Erratum ibid. 04 (2020) 202] [arXiv: 1910.04356] [INSPIRE].

[50] S. Albayrak, C. Chowdhury and S. Kharel, On loop celestial amplitudes for gauge theory and gravity, Phys. Rev. D 102 (2020) 126020 [arXiv:2007.09338] [InSPIRE].

[51] H.A. González, A. Puhm and F. Rojas, Loop corrections to celestial amplitudes, Phys. Rev. D 102 (2020) 126027 [arXiv:2009.07290] [INSPIRE].

[52] E. Casali and A. Puhm, Double Copy for Celestial Amplitudes, Phys. Rev. Lett. 126 (2021) 101602 [arXiv:2007.15027] [INSPIRE].

[53] E. Casali and A. Sharma, Celestial double copy from the worldsheet, JHEP 05 (2021) 157 [arXiv: 2011.10052] [INSPIRE].

[54] S. Banerjee, S. Ghosh and R. Gonzo, BMS symmetry of celestial OPE, JHEP 04 (2020) 130 [arXiv: 2002.00975] [INSPIRE].

[55] S. Banerjee, S. Ghosh and P. Paul, MHV graviton scattering amplitudes and current algebra on the celestial sphere, JHEP 02 (2021) 176 [arXiv:2008.04330] [INSPIRE].

[56] S. Banerjee and S. Ghosh, MHV gluon scattering amplitudes from celestial current algebras, JHEP 10 (2021) 111 [arXiv:2011.00017] [INSPIRE].

[57] M. Campiglia and J. Peraza, Generalized BMS charge algebra, Phys. Rev. D 101 (2020) 104039 [arXiv: 2002 .06691] [INSPIRE].

[58] A. Ball, E. Himwich, S.A. Narayanan, S. Pasterski and A. Strominger, Uplifting AdS $S_{3} / C F T_{2}$ to flat space holography, JHEP 08 (2019) 168 [arXiv: 1905.09809] [INSPIRE]. 
[59] A. Laddha, S.G. Prabhu, S. Raju and P. Shrivastava, The Holographic Nature of Null Infinity, SciPost Phys. 10 (2021) 041 [arXiv: 2002. 02448] [INSPIRE].

[60] L. Donnay, S. Pasterski and A. Puhm, Asymptotic Symmetries and Celestial CFT, JHEP 09 (2020) 176 [arXiv: 2005.08990] [INSPIRE].

[61] A. Atanasov, W. Melton, A.-M. Raclariu and A. Strominger, Conformal Block Expansion in Celestial CFT, arXiv:2104.13432 [INSPIRE].

[62] A. Atanasov, A. Ball, W. Melton, A.-M. Raclariu and A. Strominger, (2, 2) Scattering and the celestial torus, JHEP 07 (2021) 083 [arXiv: 2101.09591] [INSPIRE].

[63] Z.-Z. Li, H.-H. Lin and S.-Q. Zhang, Infinite Soft Theorems from Gauge Symmetry, Phys. Rev. D 98 (2018) 045004 [arXiv: 1802.03148] [INSPIRE].

[64] Y. Hamada and G. Shiu, Infinite Set of Soft Theorems in Gauge-Gravity Theories as Ward-Takahashi Identities, Phys. Rev. Lett. 120 (2018) 201601 [arXiv:1801.05528] [INSPIRE].

[65] A. Laddha and P. Mitra, Asymptotic Symmetries and Subleading Soft Photon Theorem in Effective Field Theories, JHEP 05 (2018) 132 [arXiv:1709.03850] [INSPIRE].

[66] H. Elvang, C.R.T. Jones and S.G. Naculich, Soft Photon and Graviton Theorems in Effective Field Theory, Phys. Rev. Lett. 118 (2017) 231601 [arXiv: 1611.07534] [InSPIRE].

[67] T. He, P. Mitra and A. Strominger, 2D Kac-Moody Symmetry of 4D Yang-Mills Theory, JHEP 10 (2016) 137 [arXiv: 1503. 02663] [InSPIRE].

[68] B. Czech, L. Lamprou, S. McCandlish, B. Mosk and J. Sully, A Stereoscopic Look into the Bulk, JHEP 07 (2016) 129 [arXiv:1604.03110] [INSPIRE].

[69] S. Ebert, A. Sharma and D. Wang, Descendants in celestial CFT and emergent multi-collinear factorization, JHEP 03 (2021) 030 [arXiv:2009.07881] [INSPIRE].

[70] S. Pasterski, S.-H. Shao and A. Strominger, Flat Space Amplitudes and Conformal Symmetry of the Celestial Sphere, Phys. Rev. D 96 (2017) 065026 [arXiv:1701.00049] [InSPIRE].

[71] S. Pasterski and S.-H. Shao, Conformal basis for flat space amplitudes, Phys. Rev. D 96 (2017) 065022 [arXiv:1705.01027] [INSPIRE].

[72] N. Arkani-Hamed, M. Pate, A.-M. Raclariu and A. Strominger, Celestial amplitudes from UV to IR, JHEP 08 (2021) 062 [arXiv: 2012.04208] [INSPIRE].

[73] P. Di Francesco, P. Mathieu and D. Senechal, Conformal Field Theory, Graduate Texts in Contemporary Physics, Springer-Verlag, New York (1997), [DOI] [INSPIRE].

[74] H. Kawai, D.C. Lewellen and S.H.H. Tye, A Relation Between Tree Amplitudes of Closed and Open Strings, Nucl. Phys. B 269 (1986) 1 [INSPIRE].

[75] J. Polchinski, String theory. Vol. 1: An introduction to the bosonic string, Cambridge Monographs on Mathematical Physics, Cambridge University Press (2007) [DOI] [InSPIRE]. 